\title{
COMMERCIAL EYE DROPS TRIGGERS NECROTIC EFFECT IN Acanthamoeba sp.
}

\author{
FATIMAH HASHIM ${ }^{1 *}$, NUR A'FIEFAH BINTI MOHD ZULKEFFLI ${ }^{1}$, \\ ZAFIRAH NAJWA ZAINAL ABIDIN ${ }^{1}$, MUHAMAD FAIRUS NOOR HASSIM ${ }^{1,2}$ \\ and RAMESH KUMAR SANTHANAM ${ }^{1}$ \\ ${ }^{1}$ Biological Security and Sustainability Research Group, Faculty of Science and Marine Environment, \\ Universiti Malaysia Terengganu, \\ 21030 Kuala Nerus, Terengganu, Malaysia \\ ${ }^{2}$ Institute of Tropical Biodiversity and Sustainable Development, Universiti Malaysia Terengganu, \\ 21030, Kuala Nerus TerengganuUniversiti Malaysia Terengganu, \\ 21030 Kuala Nerus, Terengganu, Malaysia \\ *E-mail: fatimah.h@umt.edu.my
}

Accepted 12 November 2020, Published online 25 December 2020

\begin{abstract}
Eye infection due to microbial infection is hard to treat and painful. Two types of eye drops that are commonly used to relieve eye pain offered by pharmacists have been tested in this study namely solution A (containing tetrahydrozoline hydrochloride) and solution B (containing gentamicin and dexamethasone). The efficacy and the activities of these eye drops were tested on Acanthamoeba sp. (a clinical isolate from an Acanthamoeba keratitis patient) to study the cytotoxicity effects of the solutions on the Acanthamoeba. The Acanthamoeba were exposed to solution A and B for $24 \mathrm{hr}$ and cell viability was assessed using MTT assays, morphological changes using the light microscope and through acridine orange and propidium iodide (AO/PI) staining for cytoplasmic biochemical activities. The $\mathrm{IC}_{50}$ value for Acanthamoeba cell viability was $45.1 \%$ and $20.3 \%$ for solutions A and B respectively. Morphological observation shows the inhibition of acanthapodia formation on the surface of the cells. Solution A and B-treated Acanthamoeba appeared in the red color of the cytoplasm upon staining with $\mathrm{AO} / \mathrm{PI}$ indicating a necrotic mode of cell death. This is due to loss of membrane integrity of Acanthamoeba cell membrane after exposed to solution $\mathrm{A}$ and $\mathrm{B}$ at their $\mathrm{IC}_{50}$ value. It is shown that solutions $\mathrm{A}$ and $\mathrm{B}$ can cause cell death in trophozoite of Acanthamoeba cells at moderate $\mathrm{IC}_{50}$ value. Unfortunately, the necrosis mode of cell death is not a preferable type of cell death for treating Acanthamoeba infection. Therefore, it can be concluded that solutions A and B are not suitable to treat eye infected with Acanthamoeba sp. as it does not promise absolute healing as the solution concentration needed is quite high and the cell death mechanism is necrosis.
\end{abstract}

Key words: Ophthalmology, gentamicin, necrosis, eye infection, Acanthamoeba keratitis

\section{INTRODUCTION}

Nowadays several drugs are available in the market to treat microbial infections in the eye. However, their efficacy is not promising due to the constituents present in it. They might be efficient against targeting the microorganism of the eye, however, it could affect the corneal layer of the host at the same time. The death of the microorganisms and debris released by the dead particles could cause negative consequences against the safety of the eyes. Generally, the microorganisms that cause infections to the eyes are viruses, bacteria, fungi, and even

\footnotetext{
* To whom correspondence should be addressed.
}

protozoa. In this study, we aimed to determine the effectiveness of two types of commercial eye drops that are readily available in the market against the protozoa phylum, Acanthamoeba species. Acanthamoeba sp. rarely infects the eye but it can cause blindness if it enters the eye. This might occur due to the use of contaminated water for face wash and contact lenses. The largest case of Acanthamoeba keratitis is mainly from the infection caused by the use of contact lenses. Currently, Polyhexamethylene biguanide (PHMB) and chlorhexidine have been used to treat Acanthamoeba keratitis (Lorenzo-Morales et al., 2015). Though these drugs are effective, results revealed that most of the commercial drugs end up 
with serious microbial keratitis. Microbial keratitis is the infection caused by microorganisms such as bacteria, fungi in the corneal layer due to infections. This type of adverse effect indicated that the current treatments are not completely successful (Seal et al., 2003; Lim et al., 2008). To avoid these limitations, the mode of action of these available drugs against the microorganisms should be inquired and clarified. Therefore in this study, we tested two commercial eye drops namely Solution A containing (tetrahydrozoline hydrochloride) and Solution B (containing gentamicin and dexamethasone acetate) to assess theirs in vitro effects against Acanthamoeba sp. based on the dose-response and the mode of cell death determination on the infective stage of Acanthamoeba sp.

\section{MATERIALS AND METHODS}

\section{Preparation of culture media}

Protease yeast glucose (PYG) media was prepared to culture the Acanthamoeba. Protease and Yeast $(3.75 \mathrm{~g})$ and the $\mathrm{D}+$ glucose $(7.5 \mathrm{~g})$ were weighed and added into Page amoeba saline solution (PAS) solution. Finally, distilled water was added to the solution to make up 1liter.

\section{Cultivation of Acanthamoeba sp.}

Acanthamoeba cells (isolated from Hospital Kuala pur) were cultured in a T-25 tissue culture flask that contains $10 \mathrm{~mL}$ of Protease-Yeast Glucose media. Then, the cells were kept in the incubator at $30^{\circ} \mathrm{C}$ to reach confluence and subcultured further for every 4 days.

\section{Determination of IC $_{\mathbf{5 0}}$ value by using MTT assay}

Acanthamoeba $\mathrm{sp}$. $\left(1 \times 10^{4}\right.$ cell/well $)$ was seeded into a 96-well microplate and incubated at $30^{\circ} \mathrm{C}$. After $24 \mathrm{hr}$ of incubation time, the culture media was removed and replaced with culture media containing Solution A and solution B at various concentration ranges from $0.7 \%$ to $60 \%$ with five replicates for each solution. After $24 \mathrm{hr}, 20 \mu \mathrm{L}$ of MTT solution $(0.2 \mathrm{mg} / \mathrm{mL})$ was added into each well and incubated for another $4 \mathrm{hr}$ at $30^{\circ} \mathrm{C}$. Then, DMSO was added to dissolve the purple formazan crystal and the absorbances of the samples were measured using a microplate ELISA reader at $570 \mathrm{~nm}$. The $\mathrm{IC}_{50}$ value was derived from the plotted graph, cell viability versus concentration of solution $\mathrm{A}$ and $\mathrm{B}$.

\section{Morphological observation after treatment using a light microscope \\ Morphological changes in Acanthamoeba sp. were observed directly under an inverted light microscope via cell treatment with $3 \mathrm{~mL}$ of Solution $\mathrm{A}$ and $\mathrm{B}$ at their $\mathrm{IC}_{50}$ value concentration in 6-well}

plates. After $24 \mathrm{hr}$ of treatment, the changes were recorded.

\section{Determination of mode of cell death}

Determinations on the mode of cell death on Acanthamoeba were based on Nakisah et al. (2012) and was carried out in 6-well plates via treating the Acanthamoeba cells $\left(1 \times 10^{4} / \mathrm{mL}\right)$ with Solution $\mathrm{A}$ and $\mathrm{B}$ at their $\mathrm{IC}_{50}$ value concentration at $30^{\circ} \mathrm{C}$ for $24 \mathrm{hr}$. Then, the untreated and treated Acanthamoeba sp. were centrifuged at $3000 \mathrm{rpm}$ for $15 \mathrm{~min}$. Later, the supernatants were discarded and the pellets were rinsed with PBS solution. Next, the pellets were resuspended with acridine orange/ propidium iodide (AO/PI) solution. The cell suspensions were incubated in the dark for $10 \mathrm{~min}$ and placed onto a slide and covered with a coverslip. Finally, the slides were visualized using a fluorescence microscope (Olympus IX50, USA) at 400 magnification time.

\section{RESULTS}

\section{IC $_{50}$ determination by using MTT assay}

Initially, the toxic effect of the solution $\mathrm{A}$ and B were tested against Acanthamoeba sp. through MTT assay and their 50\% inhibition concentration $\left(\mathrm{IC}_{50}\right)$ values were obtained. Figure 1 shows the
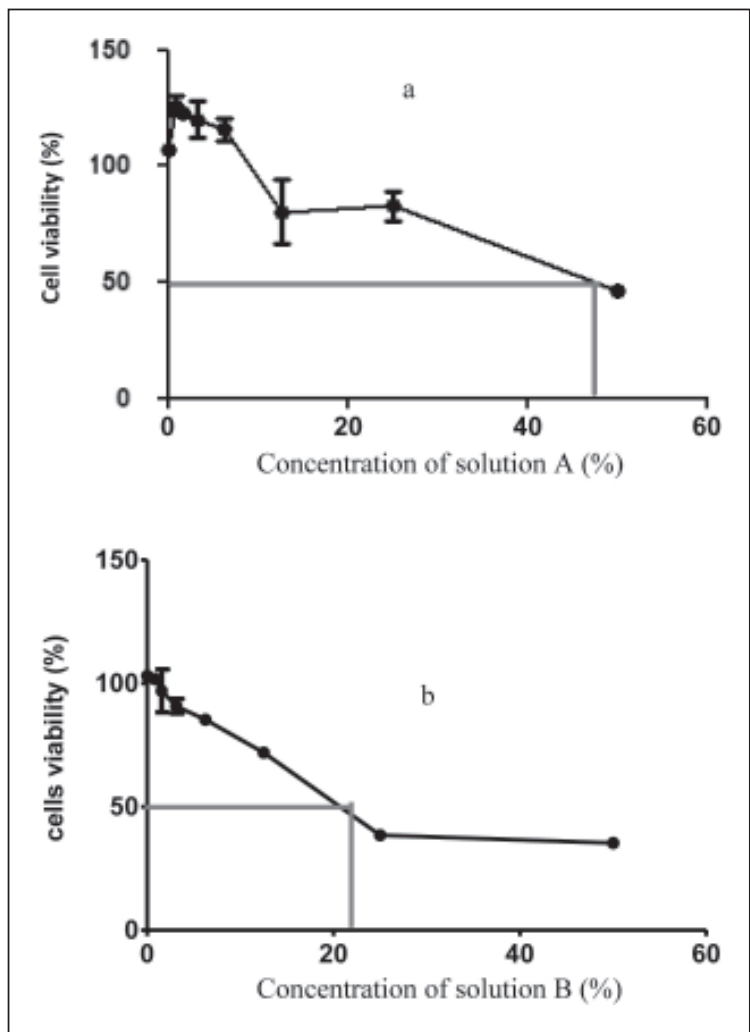

Fig. 1. Percentage of cell viability using MTT assay after 24 $\mathrm{hr}$ after the treatment of Solution $\mathrm{A}(\mathrm{a})$ and B (b). The $\mathrm{IC}_{50}$ value obtained is $45.1(\%)$ and $20.5(\%)$ Mean \pm SEM, $n=5$. 
percentage of Acanthamoeba sp. cell viability against the various concentration of Solution A and $\mathrm{B}$ with the $\mathrm{IC}_{50}$ values. The $\mathrm{IC}_{50}$ values of Solution A and B were $45.1 \%$ and $20.3 \%$, respectively. The combination of gentamicin and dexamethasone exhibits more toxicity (in vitro) towards Acanthamoeba cells in comparison with solution A that contains tetrahydrozoline hydrochloride.

\section{Morphological changes observation on Acantha-} moeba sp. treated with solution A and solution B

After $24 \mathrm{hr}$ of treatment with Solution A and B, significant morphological changes were observed on the Acanthamoeba sp. To distinguish the differences between the morphological changes that occurred in Acanthamoeba sp., the treated Acanthamoeba sp. were compared with the untreated cells which serve as a negative control. Figure 2 shows the comparison of morphological changes between untreated (Figure 2a) and treated Acanthamoeba sp. (Figures $2 \mathrm{~b} \& 2 \mathrm{c}$ ) observed under the inverted microscope. Based on the observation, the untreated cells were in trophozoite shape with acanthapodia, nucleus, and vacuoles, however, the shape of the cells treated with Solution $\mathrm{A}$ and $\mathrm{B}$ were round and cystic shape.

\section{Membrane integrity and mode of cell death determination on Acanthamoeba sp. by AO/PI staining}

The observation of the stained cells under the fluorescent microscope was used to determine the cell viability based on the membrane integrity and lysosome activities that occurred on the Acanthamoeba cells. AO/PI stained Acanthamoeba cells after exposure with the Solution A and B treatment were viewed under the fluorescent microscope (Figure 3). Compared to untreated cells, the treated Acanthamoeba cells underwent membrane disintegration and leakage occurs that leads the PI to enter into the cytoplasmic region. The green color represents the healthy cells whereas the red one represents the Acanthamoeba cells that undergo necrosis.

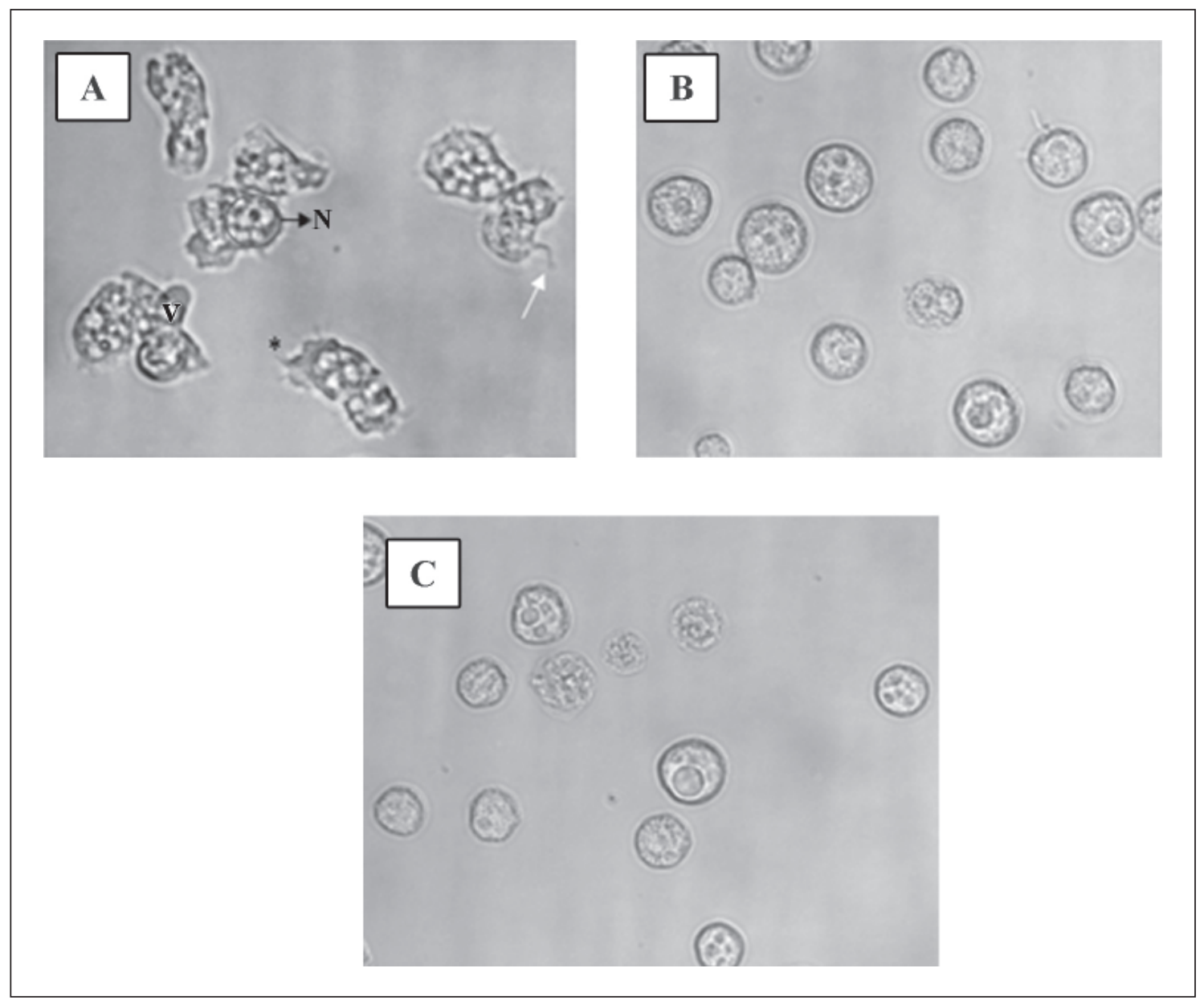

Fig. 2. Image of morphological structures of Acanthamoeba sp. under the inverted light microscope observation where (A) untreated cells show nucleus structure (N), distinctive acanthopodia (white arrow), pseudopodia (asterisk), and numerous vacuole $(\mathrm{V})$ can be seen and (B and C) treated cells with the Solution A and B can be seen with the rounded shape of a cell indicates the encystment process from its trophozoite stage. 


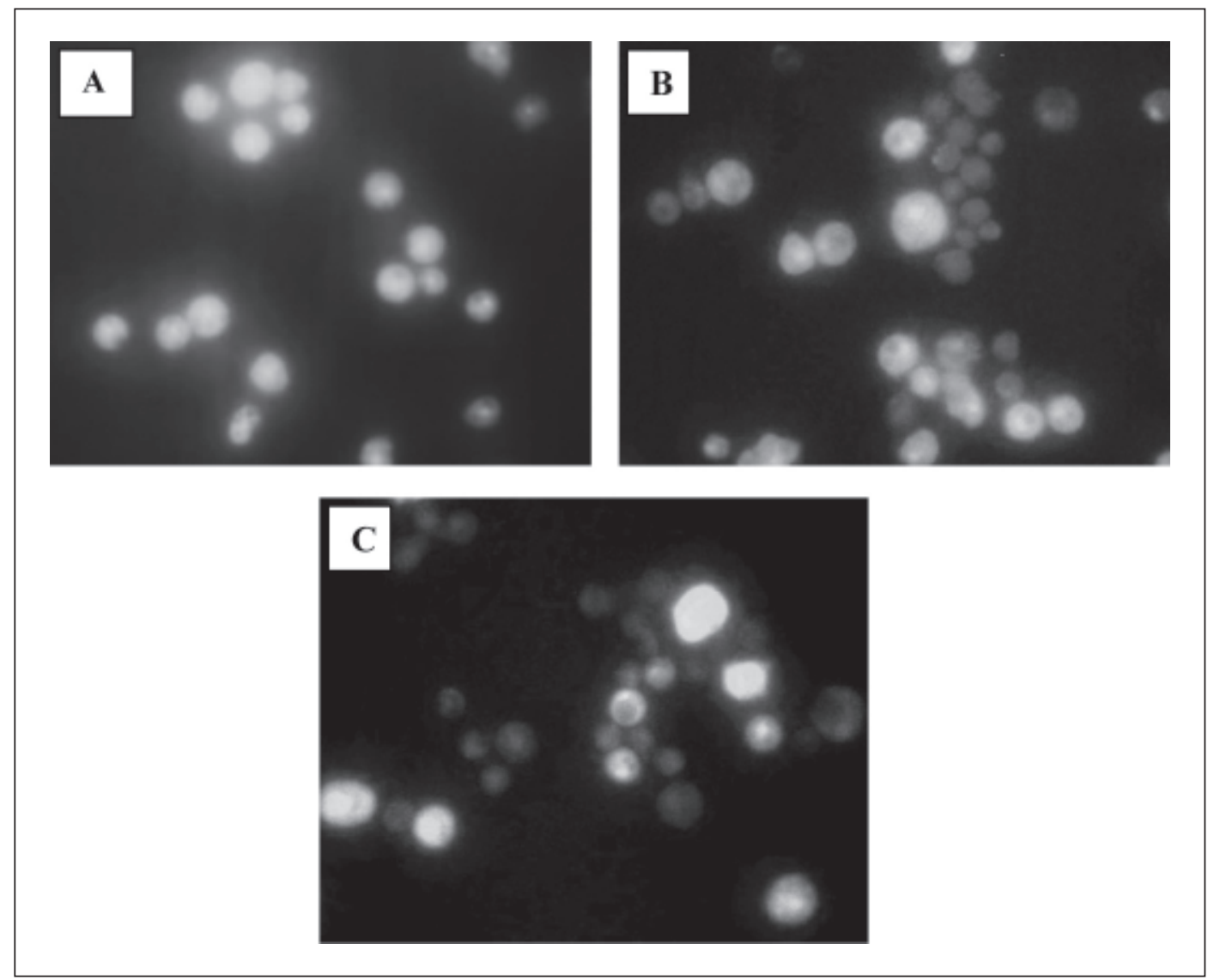

Fig. 3. The image of stained Acanthamoeba sp. after been observed under the fluorescent microscope where (A) is the untreated cells display the green cytoplasm (B and C) the treated cells appeared with the greenyellow nuclei after $24 \mathrm{hr}$ incubation time. Magnification was 400X.

\section{DISCUSSION}

Cell viability, cytotoxicity, and fifty percent inhibition concentration ( $\mathrm{IC}_{50}$ ) on Acanthamoeba sp. determination by using MTT assay

From this study, it is clear that the viability of the Acanthamoeba cells treated with Solution A and B decreased gradually as the concentration increases (Figure 1). The $\mathrm{IC}_{50}$ values for solution $\mathrm{A}$ and B were $45.1 \%$ and $20.3 \%$ respectively. Though these solutions reduced the cell viability, high $\mathrm{IC}_{50}$ values revealed that the efficacies of these samples are less against Acanthamoeba cells. Toxicity is the degree of toxic substance that may harm or affects the cells. The $\mathrm{IC}_{50}$ value is an important parameter to understand the cellular toxicity of the samples. In this study, the higher $\mathrm{IC}_{50}$ value might be due to the encystment of the Acanthamoeba cells and the inactivity of the drug (Martín-Navarro et al., 2013). The drug sensitivity testing and the development of effective therapeutic strategies might be limited due to the complexity in isolation and maintenance of the cells (Ahmad et al., 2013). The drug or compounds with lower $\mathrm{IC}_{50}$ values could be a potential complement for Acanthamoeba keratitis therapy or even first-line treatment (Martín-Navarro et al., 2013). High $\mathrm{IC}_{50}$ percentage of the solutions against Acanthamoeba sp. indicated their low efficacy in affecting the cell's viability which might not help in reducing eye infection incorporated with Acanthamoeba sp. Acanthamoeba infection produces exquisite pain and ulceration on the ocular surface resulting in cytolysis and apoptosis of the cornea tissue and thus the dissolution of corneal stroma also can occur (Clark \& Niederkorn et al., 2006).

\section{Morphological observation on Acanthamoeba sp. under the light microscopy}

The morphological changes in the Acanthamoeba cells due to the treatment of Solution A and $\mathrm{B}$ at $\mathrm{IC}_{50}$ value concentration were viewed under light microscopy. After $24 \mathrm{hr}$ of treatment with solutions $\mathrm{A}$ and $\mathrm{B}$, the morphology of the cells revealed acanthapodia and cyst characteristics. The same type of modification has not been observed in the untreated cells. It can be seen under a light microscope. 
Likewise, the untreated Acanthamoeba cells were in an inactive state, the trophozoite stage was witnessed whereas, in the treated Acanthamoeba cells, the inactive state was observed with dormant cyst and double-membrane formation. These results specified that the treated Acanthamoeba cells tended to protect them from the stress environment conditions via altering their structure (Booton et al., 2009).

The cysts are often found in the established Acanthamoeba keratitis in the corneal stroma. According to Ibrahim et al. (2014), the encystment of the Acanthamoeba cells is due to the chemical compounds. Additionally, Byers et al. (1991) reported that the occurrence of encystment is also an alternative way to develop a repetitive cycle through cell division under environmental stress or other conditions. The encystment process of Acanthamoeba cells starts with shortened acanthapodia and the formation of a double membrane. Acanthapodia is the spiny surface projections made up of hyaline cytoplasm that could penetrate the host cells. The pathogenicity of the organisms is preserved by the maintenance of the encystment rates (McClellan et al., 2001). The encystment rate is important to continue their life in a harsh environment. The irregular shape of the Acanthamoeba cells is due to the existence of the acanthapodia and the contractile vacuole. High pathogenicity will occur in the trophozoite stage which is the active stage. High pathogenicity is due to the ability of acanthapodia which adhere on the surface and invade tissues that could infect the host. The pathway of the host signalling cell will get interferes due to the binding of Acanthamoeba cells to the host (Lorenzo-Morales et al., 2015). The host cell damage is due to the role of the Acanthamoeba phospholipases as it facilitates the Acanthamoeba virulence. Also, during the adhesion process, the production of toxin secretions such as oxygen free radicals and proteases will increase and that could damage the human connective tissues. According to the findings of Marciano-Cabral and Cabral (2003), there are distributions of trophozoite and cyst in the alveoli of Granulomatous Amoebic Encephalitis (GAE) patients.

\section{Membrane permeability determination on Acanthamoeba sp. by AO/PI staining}

This paper report the first evidence on observation on the cytoplasmic activities with the effect of two eye drops on Acanthamoeba sp. Based on the results, we had confirmed the efficacies of the two eye drops on the Acanthamoeba sp. and their potential effects of using these two eye drops towards Acanthamoeba-infected eyes. Observation under fluorescence microscopy by using AO/PI revealed two important events on the Acanthamoeba cytoplasmic activities. The plasma membrane integrity and the mode of cell death either necrosis or apoptosis.

The role of the plasma membrane is to protect the cells from the surroundings, provide cell shape, and act as the selective barrier that regulates the substances which can enter and exit out from the cells. Adverse environments will disrupt the membrane integrity of the cells that could induce apoptosis, necrosis, or autophagic cells. In this study, the Acanthamoeba cells that were exposed under the stress conditions via treatment with Solution A and B for $24 \mathrm{hr}$ were stained with the combination of acridine orange and propidium iodide (AO/PI). The stained treated Acanthamoeba cells were observed under the fluorescent microscope to differentiate the viable and non-viable Acanthamoeba cells. It also helps to visualize the mode of death either apoptosis or necrosis. Acridine orange (AO) and Propidium iodide (PI) are the dyes used to detect the mode of cell death. AO is a nucleic-acid selective dye that binds to the native or denatured nucleic acid of a cell via crossing the intact membrane. Propidium iodide is a dye that cannot cross the membrane of live cells; however, it binds to the RNA.

The results obtained from the fluorescent microscope after staining is shown in Figures $3 \mathrm{~b}$ and 3c. It revealed that the Acanthamoeba cells undergo the necrosis mode of cell death. Necrosis occurs when the cells are exposed to the extreme variance from the physiological conditions that may affect the plasma membrane to damage. The plasma membrane is evoked by the agents like complement and lytic viruses due to the physiological conditions. Necrosis begins with the impairment of the cell's ability to maintain homeostasis which leads to an influx of water and extracellular ions. As a result, the intracellular cells especially the mitochondria and the entire cells will get swollen and rupture. The lysosomal enzymes will be released into the extracellular fluid due to the ultimate breakdown of the plasma membrane. The physiological significance of the necrosis process will significantly affect the group of contiguous cells. Mainly this process is evoked due to the nonphysiological disturbances and phagocytosis by the macrophages. Moreover, the effect of necrosis is the stimuli of the host inflammatory response (Choudhury et al., 2011). The inflammatory observed with extraintestinal amoebiasis is the part of the fatal contact of host cells with amoebae.

The AO/PI treated cells indicated the presence of dead cells, a necrotic type of cells. This strongly suggested that the complete damage of cells is more serious than apoptosis. Necrosis is considered to be 
the unnatural death process of cells. Necrotic cell death is often associated with extensive tissue damage and thus results in an intense inflammation response (in vivo). In this study, the orange stained organelles in the cytoplasm were appeared due to the permeable-cationic dyes that enter acidic molecules through an intact membrane. The mode of cell death that occurred on Acanthamoeba cells is also the unwanted mode of cell death, the necrosis which can induce the inflammatory effect to the host and thus results in adverse effects to the human. Thus, the efficacy of Solution A and B as the treatment to cure $\mathrm{AK}$ is weak as it induces the necrosis mode of cell death.

\section{CONCLUSION}

Overall, after $24 \mathrm{hr}$ of treatment, the two eye drops exhibited a high percentage of $\mathrm{IC}_{50}$ concentration against the Acanthamoeba sp. The morphological changes and alteration on the Acanthamoeba cell structure were observed under a fluorescence microscope through AO/PI staining that supported to determine the mode of cell death. Most of the Acanthamoeba cells after the treatment with solutions A and B underwent necrosis mode of cell death which is the undesirable type of cell death in eyes that could result in the inflammatory response to the host owing to the leakage of the chemical substances or necrotic tissues from Acanthamoeba.

\section{ACKNOWLEDGEMENTS}

This research was supported by the Faculty of Science and Marine Environment, UMT, and Final Year Project Allocation vote 401.

\section{REFERENCES}

Ahmad, A.F., Heaselgrave, W., Andrew, P.W. \& Kilvington, S. 2013. The in vitro efficacy of antimicrobial agents against the pathogenic free-living amoeba Balamuthia mandrillaris. Journal of Eukaryotic Microbiology, 60(5): 539-543.

Booton, G.C., Joslin, C.E., Shoff, M., Tu, E.Y., Kelly, D.J. \& Fuerst, P.A. 2009. Genotypic identification of Acanthamoeba sp. isolates associated with an outbreak of Acanthamoeba keratitis. Cornea, 28(6): 673-676.
Byers, T.J., Kim, B.G., King, L.E. \& Hugo, E.R. 1991. Molecular aspects of the cell cycle and encystment of Acanthamoeba. Reviews of Infectious Diseases, 13(Supplement 5): S373S384.

Choudhury, J., Kumar, S., Mayank, V., Mehta, J. \& Bardalai, D. 2011. A review on apoptosis \& its different pathway. International Journal of Biological \& Pharmaceutical Research, 3: 848861.

Clarke, D.W. \& Niederkorn, J.Y. 2006. The pathophysiology of Acanthamoeba keratitis. Trends in Parasitology, 22(4): 175-180.

Ibrahim, M.A., Yusof, M.S.M. \& Amin, N.M. 2014. Anti-amoebic properties of carbonyl thiourea derivatives. Molecules, 19(4): 5191-5204.

Lim, N., Goh, D., Bunce, C., Xing, W., Fraenkel, G., Poole, T.R. \& Ficker, L. 2008. Comparison of polyhexamethylene biguanide and chlorhexidine as monotherapy agents in the treatment of Acanthamoeba keratitis. American Journal of Ophthalmology, 145(1): 130-135.

Lorenzo-Morales, J., Khan, N.A. \& Walochnik, J. 2015. An update on Acanthamoeba keratitis: dianogsis, pathogenesis and treatment. Parasite, 22: 10 .

Marciano-Cabral, F. \& Cabral, G. 2003. Acanthamoeba spp. as agents of disease in humans. Clinical Microbiology Reviews, 16(2): 273-307.

Martín-Navarro, C.M., López-Arencibia, A., Arnalich-Montiel, F., Valladares, B., Piñero, J.E. \& Lorenzo-Morales, J. 2013. Evaluation of the in vitro activity of commercially available moxifloxacin and voriconazole eye-drops against clinical strains of Acanthamoeba. Graefe's Archive for Clinical and Experimental Ophthalmology, 251(9): 2111-2117.

McClellan, K., Howard, K., Niederkorn, J.Y. \& Alizadeh, H. 2001. Effect of steroids on Acanthamoeba cysts and trophozoites. Investigative Ophthalmology and Visual Science, 42(12): 2885-2893.

Nakisah, M.A., Muryany, M.I., Fatimah, H., Fadilah, R.N., Zalilawati, M.R., Khamsah, S. \& Habsah, M. 2012. Anti-amoebic properties of a Malaysian marine sponge Aaptos sp. on Acanthamoeba castellanii. World Journal of Microbiology and Biotechnology, 28(3): $1237-$ 1244.

Seal, D. 2003. Treatment of Acanthamoeba keratitis. Expert Review of Anti-infective Therapy, 1(2): 205-208. 